PROCEEDINGS OF THE

AMERICAN MATHEMATICAL SOCIETY

Volume 138, Number 2, February 2010, Pages 615-622

S 0002-9939(09)10079-5

Article electronically published on September 9, 2009

\title{
BOUNDARY REPRESENTATIONS ON CO-INVARIANT SUBSPACES OF BERGMAN SPACE
}

\author{
WEI HE
}

(Communicated by Marius Junge)

\begin{abstract}
Let $M$ be an invariant subspace of the Bergman space $L_{a}^{2}(\mathbb{D})$ and $S_{M}$ be the compression of the coordinate multiplication operator $M_{z}$ to the co-invariant subspace $L_{a}^{2}(\mathbb{D}) \ominus M$. The present paper determines when the identity representation of $C^{*}\left(S_{M}\right)$ is a boundary representation for the Banach subalgebra $\mathcal{B}\left(S_{M}\right)$. The paper also considers boundary representations on the co-invariant subspaces of $L_{a}^{2}\left(\mathbb{B}_{n}\right)$.
\end{abstract}

\section{INTRODUCTION}

In his two Acta papers Arv1, Arv2, Arveson systematically studied relations between non-self-adjoint operator algebras on Hilbert space and $C^{*}$-algebras generated by them. One of the main purposes is to understand to what extent an algebra of operators on a Hilbert space can determine the structure of the $C^{*}$-algebra generated by it. As a typical example, Arveson investigated for an invariant subspace $M$ of the Hardy space $H^{2}(\mathbb{D})$, when the structure of the $C^{*}$-algebra generated by $S_{M}$ and the identity can be determined by the Banach subalgebra generated by $S_{M}$ and the identity, where $S_{M}$ is the compression of the coordinate multiplication operator $M_{z}$ to the co-invariant subspace $H^{2}(\mathbb{D}) \ominus M$. The present paper considers the same problem on the Bergman space over the unit disk.

For convenience, let us first introduce some notation and recall some basic definitions. For a Hilbert space $H$, let $\mathcal{L}(H)$ (resp. $\mathcal{K}(H))$ denote the set of all bounded (resp. compact) operators on $H$. For a subset $S \subseteq \mathcal{L}(H)$, let $C^{*}(S)$ be the $C^{*}$ algebra generated by $S$ and the identity, and let $\mathcal{B}(\bar{S})$ be the operator norm-closed Banach algebra generated by $S$ and the identity. Let $B_{1}, B_{2}$ be two $C^{*}$-algebras, and let $\phi$ be a linear map of $B_{1}$ into $B_{2}$. If $M_{k}, k=1,2, \ldots$, denotes the $C^{*}$-algebra of all complex $k \times k$ matrices and $i d_{k}$ denotes the identity map of $M_{k}$, then $i d_{k} \otimes \phi$ is a linear map of $M_{k} \otimes B_{1}$ into $M_{k} \otimes B_{2}$. The map $\phi$ is called completely positive (resp. completely isometric) provided that every map in the sequence $i d_{1} \otimes \phi$, $i d_{2} \otimes \phi, \ldots$ is positive (resp. isometric).

One of the principal concepts in Arveson's work [Arv1, Arv2 is that of boundary representations. Let $B$ be a $C^{*}$-algebra and let $A$ be a linear subspace of $B$ such that $B=C^{*}(A)$. An irreducible representation $\omega$ of $B$ is called a boundary representation for $A$ if $\left.\omega\right|_{A}$ has a unique completely positive linear extension to $B$,

Received by the editors March 28, 2008, and, in revised form, April 20, 2009.

2000 Mathematics Subject Classification. Primary 47L55, 46E22.

(C)2009 American Mathematical Society Reverts to public domain 28 years from publication 
namely $\omega$ itself. Boundary representations of $C^{*}$-algebras have been comprehensively investigated; see Arv1, Arv2, Arv3, Arv4, GHX for more information. In particular, if the identity representation of $B$ is a boundary representation for $A$, then the subspace $A$ can determine the structure of the $C^{*}$-algebra $B$.

Arveson explored a typical example in Arv1, Arv2. We restate his results as follows. Let $\mathbb{D}$ be the open unit disk, and let $\mathbb{T}$ be the unit circle. The Hardy space $H^{2}(\mathbb{D})$ consists of all functions in $L^{2}(\mathbb{T})$ whose negative Fourier coefficients vanish. Let $M$ be an invariant subspace of $H^{2}(\mathbb{D})$; i.e., $M$ is closed and invariant under the coordinate multiplication operator $M_{z}$. By the well known Beurling theorem, $M=\eta H^{2}(\mathbb{D})$ for some inner function $\eta$. To exclude trivial cases, suppose $\eta$ is not a Möbius transform. Let $N=H^{2}(\mathbb{D}) \ominus M$, then $N$ is co-invariant; that is, $N$ is invariant under $M_{z}^{*}$. Conversely, each co-invariant subspace has such a form. Set $S_{M}=\left.P_{N} M_{z}\right|_{N}$, where $P_{N}$ is the orthogonal projection from $H^{2}(\mathbb{D})$ to $N$. Arveson determined when the identity representation of $C^{*}\left(S_{M}\right)$ is a boundary representation for the Banach subalgebra $\mathcal{B}\left(S_{M}\right)$.

Theorem 1.1 (Arv1, Arv2]). The identity representation of $C^{*}\left(S_{M}\right)$ is a boundary representation for the subalgebra $\mathcal{B}\left(S_{M}\right)$ if and only if $Z_{\eta} \cap \mathbb{T}$ is a proper subset of $\mathbb{T}$, where $Z_{\eta}$ consists of the zeroes of $\eta$ inside $\mathbb{D}$, along with all points $\lambda$ on $\mathbb{T}$ for which $\eta$ cannot be continued analytically from $\mathbb{D}$ to $\lambda$.

The proof of this theorem is very complicated. In Arv1, Arveson only proved the extreme case $Z_{\eta} \cap \mathbb{T}=\mathbb{T}$ and $Z_{\eta} \cap \mathbb{T}$ has Lebesgue measure zero. The above general result was obtained in Arv2.

The present paper deals with the same problem on the Bergman space $L_{a}^{2}(\mathbb{D})$. Recall that the Bergman space $L_{a}^{2}(\mathbb{D})$ is the closed subspace of $L^{2}(\mathbb{D}, d A)$ consisting of analytic functions, where $d A$ is the normalized area measure on $\mathbb{D}$. For each $f \in L_{a}^{2}(\mathbb{D})$, set

$$
Z_{*}(f)=\{\lambda \in \mathbb{D}: f(\lambda)=0\} \cup\left\{\lambda \in \mathbb{T}: \liminf _{z \in \mathbb{D}, z \rightarrow \lambda}|f(z)|=0\right\} .
$$

Given an invariant subspace $M$ of $L_{a}^{2}(\mathbb{D})$, set

$$
Z_{*}(M)=\bigcap_{f \in M} Z_{*}(f) .
$$

To exclude trivial cases, we always assume that $M$ is infinite co-dimensional; that is, the co-invariant subspace $L_{a}^{2}(\mathbb{D}) \ominus M$ is infinite dimensional. Now our main result can be stated as follows.

Theorem 1.2. Let $M$ be an infinite co-dimensional invariant subspace of $L_{a}^{2}(\mathbb{D})$, $N=L_{a}^{2}(\mathbb{D}) \ominus M$ and $S_{M}=\left.P_{N} M_{z}\right|_{N}$. Then the identity representation of $C^{*}\left(S_{M}\right)$ is a boundary representation for the subalgebra $\mathcal{B}\left(S_{M}\right)$ if and only if $\operatorname{dim} M \ominus z M=1$ and $Z_{*}(M) \cap \mathbb{T}$ is a proper subset of $\mathbb{T}$.

The proof of this theorem will be presented in Section 2. One will find in the proof that the distinction between our result and that of Arveson is essentially due to the difference between the invariant subspace structure of the two spaces $L_{a}^{2}(\mathbb{D})$ and $H^{2}(\mathbb{D})$.

In Section 3, we obtain some results on boundary representations on the coinvariant subspaces of $L_{a}^{2}\left(\mathbb{B}_{n}\right)$, where $\mathbb{B}_{n}$ denotes the unit ball of $\mathbb{C}^{n}$. 


\section{Proof of the MAin Result}

In this section, we will give the proof of the main result Theorem 1.2

For an irreducible set $S$ of operators, there is a general criterion for determining whether the identity representation of $C^{*}(S)$ is a boundary representation for $S$. It is the following Arveson's boundary theorem.

Theorem 2.1 (Arv2]). Let $S$ be an irreducible set of operators on a Hilbert space $H$, such that $S$ contains the identity and $C^{*}(S)$ contains the algebra $\mathcal{K}(H)$ of all compact operators on $H$. Then the identity representation of $C^{*}(S)$ is a boundary representation for $S$ if and only if the quotient map $q: \mathcal{L}(H) \rightarrow \mathcal{L}(H) / \mathcal{K}(H)$ is not completely isometric on the linear span of $S \cup S^{*}$.

From the above theorem, Arveson obtained the following corollary which is more convenient for applications. In the following, let $\sigma(T)$ denote the spectrum of an operator $T$, and $\sigma_{e}(T)$ denote the essential spectrum of $T$. Let $\left|\sigma_{e}(T)\right|=$ $\sup \left\{|\lambda|: \lambda \in \sigma_{e}(T)\right\}$, the essential spectral radius of $T$. An operator $T$ is said to be essentially normal if the self-commutator, $\left[T^{*}, T\right]=T^{*} T-T T^{*}$, is compact.

Corollary 2.2 ([Arv2]). Let $S$ be an irreducible set of commuting essentially normal operators which contains the identity. Assume that $\left|\sigma_{e}(T)\right|<\|T\|$ for some element $T \in S$. Then the identity representation of $C^{*}(S)$ is a boundary representation for $S$.

The following result is also useful in our proof.

Lemma 2.3 ([Arv1, Theorem 3.6.3]). Let $T$ be a contraction on a Hilbert space. Then $T$ gives rise to a completely isometric representation of the disk algebra $A(\mathbb{D})$ if and only if $\sigma(T)$ contains the unit circle. For such a $T$, the identity representation of $C^{*}(T)$ is never a boundary representation for $\mathcal{B}(T)$.

We also need some results about the operator $S_{M}$.

Lemma 2.4 ([Zhu, Theorem 3.1]). Suppose $M$ is an invariant subspace of $L_{a}^{2}(\mathbb{D})$, $R_{M}=\left.M_{z}\right|_{M}, N=L_{a}^{2}(\mathbb{D}) \ominus M$, and $S_{M}=\left.P_{N} M_{z}\right|_{N}$, then the following are equivalent:

(1) $\left[R_{M}^{*}, R_{M}\right]=R_{M}^{*} R_{M}-R_{M} R_{M}^{*}$ is compact.

(2) $\left[S_{M}^{*}, S_{M}\right]=S_{M}^{*} S_{M}-S_{M} S_{M}^{*}$ is compact.

(3) $\operatorname{dim} M \ominus z M<\infty$.

Lemma 2.5 ([Ri, Corollary 4.6] and Hed, Theorem 1.3]). Let $M$ be an invariant subspace of $L_{a}^{2}(\mathbb{D}), M \neq\{0\}$, and $S_{M}$ be as in Lemma 2.4. If $\operatorname{dim} M \ominus z M=1$, then $\sigma\left(S_{M}\right)=Z_{*}(M)$. If $\operatorname{dim} M \ominus z M>1$, then $\sigma\left(S_{M}\right)=\overline{\mathbb{D}}$.

Lemma 2.6. Let $M, N$ and $S_{M}$ be as in Lemma 2.4, Then the operator $S_{M}$ is irreducible.

Before proving the lemma, let us recall the definition of the Berezin transform. Note that $L_{a}^{2}(\mathbb{D})$ is a reproducing kernel Hilbert space; i.e., for any $\lambda \in \mathbb{D}$, the evaluation functional $E_{\lambda}: f \mapsto f(\lambda)$ is continuous. By the Riesz theorem, for every $\lambda \in \mathbb{D}$, there is a unique function $K_{\lambda} \in L_{a}^{2}(\mathbb{D})$ such that

$$
f(\lambda)=\left\langle f, K_{\lambda}\right\rangle, \quad f \in L_{a}^{2}(\mathbb{D}) .
$$

$K_{\lambda}$ is called the reproducing kernel of $L_{a}^{2}(\mathbb{D})$ at $\lambda$. Direct computation shows that on the Bergman space $L_{a}^{2}(\mathbb{D}), K_{\lambda}(z)=1 /(1-\bar{\lambda} z)^{2}$. Set $k_{\lambda}=K_{\lambda} /\left\|K_{\lambda}\right\|$. For a 
bounded linear operator $A$ on $L_{a}^{2}(\mathbb{D})$, the Berezin transform of $A$ is defined by

$$
\tilde{A}(\lambda)=\left\langle A k_{\lambda}, k_{\lambda}\right\rangle, \quad \lambda \in \mathbb{D} .
$$

It is well known that for two bounded linear operators $A_{1}$ and $A_{2}$ on $L_{a}^{2}(\mathbb{D}), \tilde{A}_{1}=\tilde{A}_{2}$ if and only if $A_{1}=A_{2}$.

For two functions $f$ and $g$ in $L_{a}^{2}(\mathbb{D})$, let $f \otimes g$ be the rank-one operator defined by

$$
(f \otimes g) h=\langle h, g\rangle f, \quad h \in L_{a}^{2}(\mathbb{D}) .
$$

Proof. Since the reproducing kernel of $L_{a}^{2}(\mathbb{D})$ is $K_{\lambda}(z)=1 /(1-\bar{\lambda} z)^{2}$, it is easy to compute that

$$
\left\langle(1 \otimes 1) k_{\lambda}, k_{\lambda}\right\rangle=\left|\left\langle k_{\lambda}, 1\right\rangle\right|^{2}=1 /\left\|K_{\lambda}\right\|^{2}=\left(1-|\lambda|^{2}\right)^{2} .
$$

One can also verify that

$$
M_{z}^{*} k_{\lambda}=\bar{\lambda} k_{\lambda}
$$

and hence

$$
\begin{aligned}
& \left\langle\left(i d-2 M_{z} M_{z}^{*}+M_{z}^{2} M_{z}^{* 2}\right) k_{\lambda}, k_{\lambda}\right\rangle \\
= & \left\langle k_{\lambda}, k_{\lambda}\right\rangle-2\left\langle M_{z}^{*} k_{\lambda}, M_{z}^{*} k_{\lambda}\right\rangle+\left\langle M_{z}^{* 2} k_{\lambda}, M_{z}^{* 2} k_{\lambda}\right\rangle \\
= & \left(1-|\lambda|^{2}\right)^{2} .
\end{aligned}
$$

By the above-mentioned property of the Berezin transform, we have

$$
1 \otimes 1=i d-2 M_{z} M_{z}^{*}+M_{z}^{2} M_{z}^{* 2} .
$$

Then

$$
P_{N}(1 \otimes 1) P_{N}=P_{N}\left(i d-2 M_{z} M_{z}^{*}+M_{z}^{2} M_{z}^{* 2}\right) P_{N},
$$

and hence

$$
P_{N} 1 \otimes P_{N} 1=i d_{N}-2 S_{M} S_{M}^{*}+S_{M}^{2} S_{M}^{* 2} .
$$

Let $e=P_{N} 1$, then $e \neq 0$. Assume that there is some projection $Q$ commuting with $S_{M}$, and we will show that $Q$ is trivial. Clearly, $Q$ commutes with the right-hand side of (2.2), so it commutes with the left-hand side. That is

$$
Q(e \otimes e)=(e \otimes e) Q,
$$

and immediately,

$$
Q e \otimes e=e \otimes Q e .
$$

Therefore, $Q e=c e$ for some constant $c$. Since

$$
c e=Q e=Q(Q e)=Q(c e)=c Q e=c^{2} e
$$

and $e \neq 0$, we have $c=0$ or $c=1$.

If $c=0$, then $Q e=0$, and thus $S_{M}^{n} Q e=0$ for any integer $n$. Since $Q$ commutes with $S_{M}, Q S_{M}^{n} e=0$. That is, $Q P_{N} z^{n}=0$ for any $n$. But the linear span of $\left\{P_{N} z^{n}\right\}_{n=0}^{\infty}$ is dense in $P_{N} L_{a}^{2}(\mathbb{D})$, so we get $Q=0$.

If $c=1$, then $Q e=e$, i.e., $(1-Q) e=0$. The same argument as above gives that $1-Q=0$, and hence $Q=1$.

The above reasoning shows that there is no nontrivial projection commuting with $S_{M}$, so the operator $S_{M}$ is irreducible. This completes the proof.

Lemma 2.7. Let $M, N$, and $S_{M}$ be as in Lemma 2.4. Suppose $M$ satisfies $\operatorname{dim} M \ominus$ $z M<\infty$. Then $\sigma_{e}\left(S_{M}\right)=\sigma\left(S_{M}\right) \cap \mathbb{T}$. 
Proof. The proof is similar to Arveson's proof in the Hardy space case (cf. Arv1]). Let $\operatorname{Comm}\left(S_{M}\right)$ be the commutator ideal of $C^{*}\left(S_{M}\right)$, i.e., the closed ideal generated by $\left\{x y-y x: x, y \in C^{*}\left(S_{M}\right)\right\}$. Since $\operatorname{dim} M \ominus z M<\infty$, it follows from Lemma 2.4 that

$$
\left[S_{M}^{*}, S_{M}\right]=S_{M}^{*} S_{M}-S_{M} S_{M}^{*} \in \mathcal{K} .
$$

Lemma 2.6 says that the operator $S_{M}$ is irreducible, so $\operatorname{Comm}\left(S_{M}\right)=\mathcal{K}$ (see for example [Arv1, Corollary 3.3.7]).

Since $C^{*}\left(S_{M}\right) / \operatorname{Comm}\left(S_{M}\right)=C^{*}\left(S_{M}\right) / \mathcal{K}$ is an abelian $C^{*}$-algebra, there is a natural bijection between the characters of $C^{*}\left(S_{M}\right) / \operatorname{Comm}\left(S_{M}\right)$ and the points in $\sigma_{e}\left(S_{M}\right)$. At the same time, since a character of $C^{*}\left(S_{M}\right)$ must vanish on $\operatorname{Comm}\left(S_{M}\right)$, there is also a natural bijection between characters of $C^{*}\left(S_{M}\right)$ and characters of $C^{*}\left(S_{M}\right) / \operatorname{Comm}\left(S_{M}\right)$. Therefore, the characters of $C^{*}\left(S_{M}\right)$ are in one-to-one correspondence with points in $\sigma_{e}\left(S_{M}\right)$.

Let $\Gamma: \chi \mapsto \chi\left(S_{M}\right)$ be the canonical bijective map from the characters of $C^{*}\left(S_{M}\right)$ to the points of $\sigma_{e}\left(S_{M}\right)$. We will show that $\Gamma$ is also a bijection from the characters of $C^{*}\left(S_{M}\right)$ to the points of $\sigma\left(S_{M}\right) \cap \mathbb{T}$, by which the conclusion will follow.

Now if $\lambda$ is a point in $\sigma\left(S_{M}\right) \cap \mathbb{T}$, then $|\lambda|=1=\left\|S_{M}\right\|$, so $\lambda \in \partial W\left(S_{M}\right)$, where $W\left(S_{M}\right)=\left\{\left\langle S_{M} \xi, \xi\right\rangle: \xi \in N,\|\xi\|=1\right\}$ is the numerical range of $S_{M}$. By Arv1, Theorem 3.1.2], there is a unique character $\chi$ of $C^{*}\left(S_{M}\right)$ such that $\chi\left(S_{M}\right)=\lambda$.

Conversely, suppose $\chi$ is a character of $C^{*}\left(S_{M}\right)$ and we must show that $\chi\left(S_{M}\right) \in$ $\sigma\left(S_{M}\right) \cap \mathbb{T}$. But $\chi\left(S_{M}\right) \in \sigma\left(S_{M}\right)$ is obvious, so it remains to show $\left|\chi\left(S_{M}\right)\right|=1$. Since $\chi$ vanishes on $\operatorname{Comm}\left(S_{M}\right)=\mathcal{K}$, applying $\chi$ to formula (2.2) gives

$$
1-2\left|\chi\left(S_{M}\right)\right|^{2}+\left|\chi\left(S_{M}\right)\right|^{4}=0
$$

and hence $\left|\chi\left(S_{M}\right)\right|=1$.

We are now ready to prove the main result Theorem 1.2

Proof of Theorem 1.2. The necessity part. We need to show that if the identity representation of $C^{*}\left(S_{M}\right)$ is a boundary representation for the subalgebra $\mathcal{B}\left(S_{M}\right)$, then $\operatorname{dim} M \ominus z M=1$ and $Z_{*}(M) \cap \mathbb{T}$ is a proper subset of $\mathbb{T}$.

Since $\left\|S_{M}\right\| \leq\left\|M_{z}\right\|=1, S_{M}$ is a contraction. Suppose $\operatorname{dim} M \ominus z M>1$. Then by Lemma 2.5. we have $\sigma\left(S_{M}\right)=\overline{\mathbb{D}} \supseteq \mathbb{T}$. Then Lemma 2.3 implies that the identity representation of $C^{*}\left(S_{M}\right)$ is not a boundary representation for the subalgebra $\mathcal{B}\left(S_{M}\right)$, a contradiction. Hence $\operatorname{dim} M \ominus z M=1$.

Suppose $Z_{*}(M) \cap \mathbb{T}$ is not a proper subset of $\mathbb{T}$, i.e., $Z_{*}(M) \cap \mathbb{T}=\mathbb{T}$. Since we have just proved $\operatorname{dim} M \ominus z M=1$, it follows from Lemma 2.5 that $\sigma\left(S_{M}\right)=Z_{*}(M) \supseteq \mathbb{T}$. Using Lemma 2.3 again, we get a contradiction.

The sufficiency part. Suppose $\operatorname{dim} M \ominus z M=1$ and $Z_{*}(M) \cap \mathbb{T}$ is a proper subset of $\mathbb{T}$, we will prove that the identity representation of $C^{*}\left(S_{M}\right)$ is a boundary representation for the subalgebra $\mathcal{B}\left(S_{M}\right)$.

Since $\operatorname{dim} M \ominus z M=1$, by Lemma 2.4, the operator $S_{M}^{*} S_{M}-S_{M} S_{M}^{*}$ is compact. Lemma 2.6 says that the operator $S_{M}$ is irreducible. So according to Corollary 2.2. it suffices to show that if $Z_{*}(M) \cap \mathbb{T}$ is a proper subset of $\mathbb{T}$, then there is a polynomial $p$ such that

$$
\left|\sigma_{e}\left(p\left(S_{M}\right)\right)\right|=\sup \left\{|p(\lambda)|: \lambda \in \sigma_{e}\left(S_{M}\right)\right\}<\left\|p\left(S_{M}\right)\right\| .
$$


The following proof uses the same idea as that in Arv2, Theorem 2.2.1, Corollary 1]. Since $M$ satisfies $\operatorname{dim} M \ominus z M=1$, by Lemma 2.7 ,

$$
\sigma_{e}\left(S_{M}\right)=\sigma\left(S_{M}\right) \cap \mathbb{T}=Z_{*}(M) \cap \mathbb{T} .
$$

If we can show that $Z_{*}(M) \cap \mathbb{T}$ is not a spectral set for $S_{M}$, then the conclusion follows. Suppose $S_{M}$ has $Z_{*}(M) \cap \mathbb{T}$ as its spectral set. Since the complement of $Z_{*}(M) \cap \mathbb{T}$ is connected and $Z_{*}(M) \cap \mathbb{T}$ has no interior, $S_{M}$ must be normal, a contradiction. The proof is complete.

Remark 2.8. We have completely determined when the identity representation of $C^{*}\left(S_{M}\right)$ is a boundary representation for the subalgebra $\mathcal{B}\left(S_{M}\right)$. One may wonder for an invariant subspace $M$ of $L_{a}^{2}(\mathbb{D})$, when the identity representation of $C^{*}\left(R_{M}\right)$ is a boundary representation for the subalgebra $\mathcal{B}\left(R_{M}\right)$, where $R_{M}=\left.M_{z}\right|_{M}$. This is an easy consequence of [GHX, Theorem 3.2] which says that if $T$ is subnormal and $\sigma(T)=\overline{\mathbb{D}}$, then the identity representation of $C^{*}(T)$ is not a boundary representation for the subalgebra $\mathcal{B}(T)$. Since $R_{M}$ is subnormal and $\sigma\left(R_{M}\right)=\overline{\mathbb{D}}$ [Zhu, Proposition 2.1], we know that the identity representation of $C^{*}\left(R_{M}\right)$ is never a boundary representation for the subalgebra $\mathcal{B}\left(R_{M}\right)$.

\section{Results on Co-invariant subspaces of $L_{a}^{2}\left(\mathbb{B}_{n}\right)$}

In this section, we will consider boundary representations on co-invariant subspaces of the Bergman space $L_{a}^{2}\left(\mathbb{B}_{n}\right)$.

Recall that the Bergman space $L_{a}^{2}\left(\mathbb{B}_{n}\right)$ is the subspace of $L^{2}\left(\mathbb{B}_{n}, d V\right)$ consisting of analytic functions over the unit ball $\mathbb{B}_{n}$, where $d V$ is the normalized volume measure. Let $M$ be an invariant subspace of $L_{a}^{2}\left(\mathbb{B}_{n}\right)$, i.e., $M$ is closed and invariant under the coordinate multiplication operators $\left\{M_{z_{1}}, \ldots, M_{z_{n}}\right\}$. Let $N=L_{a}^{2}\left(\mathbb{B}_{n}\right) \ominus$ $M$ be the co-invariant subspace and $S_{i}=\left.P_{N} M_{z_{i}}\right|_{N}$ for $1 \leq i \leq n$.

An interesting problem is to characterize all the boundary representations of $C^{*}\left(S_{1}, \ldots, S_{n}\right)$ relative to $\mathcal{B}\left(S_{1}, \ldots, S_{n}\right)$, where $\mathcal{B}\left(S_{1}, \ldots, S_{n}\right)$ is the operator normclosed Banach algebra generated by $S_{1}, \ldots, S_{n}$ and the identity. In particular, we are mainly concerned with whether the identity representation of $C^{*}\left(S_{1}, \ldots, S_{n}\right)$ is a boundary representation for the subalgebra $\mathcal{B}\left(S_{1}, \ldots, S_{n}\right)$. This problem has a close connection with the essential normality of the operator tuple $\left(S_{1}, \ldots, S_{n}\right)$. Recall that the operator tuple $\left(S_{1}, \ldots, S_{n}\right)$ is said to be essentially normal if $S_{i}^{*} S_{j}-S_{j} S_{i}^{*}$ is compact for all $1 \leq i, j \leq n$. For brevity, we often say that the co-invariant subspace $N=L_{a}^{2}\left(\mathbb{B}_{n}\right) \ominus M$ on which the operator tuple $\left(S_{1}, \ldots, S_{n}\right)$ acts is essentially normal. In an even more general setting than the Bergman space $L_{a}^{2}\left(\mathbb{B}_{n}\right)$, essential normality has been explored deeply. See for example Arv5, Dou, Guo, GW1, GW2, GWa.

The results on essential normality shed some light on the problem of boundary representations. First of all, it is an empirical fact that on many Hilbert spaces of analytic functions, the operator tuple $\left(S_{1}, \ldots, S_{n}\right)$ is irreducible. If the tuple is also essentially normal, then $C^{*}\left(S_{1}, \ldots, S_{n}\right)$ must contain the algebra $\mathcal{K}$ of all compact operators. As a result of this, we can use Arveson's boundary theorem, Theorem 2.1. to tell us whether the identity representation of $C^{*}\left(S_{1}, \ldots, S_{n}\right)$ is a boundary representation for the subalgebra $\mathcal{B}\left(S_{1}, \ldots, S_{n}\right)$. The following theorem is an attempt in this direction.

Theorem 3.1. Let $p$ be a homogeneous polynomial, and let $M=[p]$ be the invariant subspace of $L_{a}^{2}\left(\mathbb{B}_{n}\right)$ generated by $p$. Let $p=p_{1}^{k_{1}} \cdots p_{l}^{k_{l}}$ be the irreducible factorization 
of $p$. Suppose there is some $k_{i}$ such that $k_{i}>1$, then the identity representation of $C^{*}\left(S_{1}, \ldots, S_{n}\right)$ is a boundary representation for the subalgebra $\mathcal{B}\left(S_{1}, \ldots, S_{n}\right)$.

Proof. It is shown in GW1] that for an invariant subspace $M$ of $L_{a}^{2}\left(\mathbb{B}_{n}\right)$ which is generated by a homogeneous polynomial $p$, the co-invariant subspace $L_{a}^{2}\left(\mathbb{B}_{n}\right) \ominus M$ is essentially normal; i.e., $S_{i}^{*} S_{j}-S_{j} S_{i}^{*}$ is compact for all $1 \leq i, j \leq n$. The same paper also shows that the operator tuple $\left(S_{1}, \ldots, S_{n}\right)$ is irreducible, and the essential spectrum is $\sigma_{e}\left(S_{1}, \ldots, S_{n}\right)=Z(p) \cap \partial \mathbb{B}_{n}$, so there is a short exact sequence

$$
0 \rightarrow \mathcal{K} \rightarrow C^{*}\left(S_{1}, \ldots, S_{n}\right) \rightarrow C\left(Z(p) \cap \partial \mathbb{B}_{n}\right) \rightarrow 0,
$$

where $Z(p)$ is the zero set of $p$ and $C\left(Z(p) \cap \partial \mathbb{B}_{n}\right)$ is the set of continuous functions on $Z(p) \cap \partial \mathbb{B}_{n}$. Consider the operator $S_{q}$ with $q=p_{1} \cdots p_{l}$, then $S_{q} \neq 0$. Since $\left.q\right|_{Z(p) \cap \partial \mathbb{B}_{n}}=0, S_{q}$ is compact by the exactness of the above sequence. Hence the map

$$
q: \mathcal{L}(N) \rightarrow \mathcal{L}(N) / \mathcal{K}(N)
$$

is not completely isometric on the linear span of $\mathcal{B}\left(S_{1}, \ldots, S_{n}\right) \cup \mathcal{B}\left(S_{1}^{*}, \ldots, S_{n}^{*}\right)$. Applying Arveson's boundary theorem, Theorem 2.1, we obtain the desired conclusion.

Remark 3.2. As in the $L_{a}^{2}(\mathbb{D})$ case, one naturally asks for an invariant subspace $M$ of $L_{a}^{2}\left(\mathbb{B}_{n}\right)$, when the identity representation of $C^{*}\left(R_{1}, \ldots, R_{n}\right)$ is a boundary representation for the subalgebra $\mathcal{B}\left(R_{1}, \ldots, R_{n}\right)$, where $R_{i}=\left.M_{z_{i}}\right|_{M}$ for $1 \leq i \leq n$. The operator tuple $\left(R_{1}, \ldots, R_{n}\right)$ is obviously subnormal. When $M$ is homogeneous (i.e., $M$ is generated by homogeneous polynomials) and essentially normal (i.e., the operator $R_{i} R_{j}^{*}-R_{j}^{*} R_{i}$ is compact for all $\left.1 \leq i, j \leq n\right), \sigma\left(R_{1}, \ldots, R_{n}\right)=$ $\overline{\mathbb{B}}_{n}$ by GW1. Consequently, if $M$ is homogeneous and essentially normal, the identity representation of $C^{*}\left(R_{1}, \ldots, R_{n}\right)$ is never a boundary representation for the subalgebra $\mathcal{B}\left(R_{1}, \ldots, R_{n}\right)$ by [GHX, Theorem 3.2].

Remark 3.3. The results in this paper also hold on the weighted Bergman space without modifying the proofs.

\section{REFERENCES}

[Arv1] W. Arveson, Subalgebras of $C^{*}$-algebras, Acta Math. 123(1969), 141-224. MR0253059 (40:6274)

[Arv2] W. Arveson, Subalgebras of $C^{*}$-algebras II, Acta Math. 128(1972), 271-308. MR0394232 $(52: 15035)$

[Arv3] W. Arveson, Subalgebras of $C^{*}$-algebras III: Multivariable operator theory, Acta Math. 181(1998), 159-228. MR.1668582 (2000e:47013)

[Arv4] W. Arveson, The noncommutative Choquet boundary, J. Amer. Math. Soc. 21(2008), 1065-1084. MR2425180 (2009g:46108)

[Arv5] W. Arveson, p-summable commutators in dimension d, J. Operator Theory 54(2005), 101-117. MR 2168861 (2007b:47012)

[Dou] R. Douglas, Essentially reductive Hilbert modules, J. Operator Theory 55(2006), 117-133. MR2212024 (2007h:47014)

[Guo] K. Guo, Defect operators for submodules of $H_{d}^{2}$, J. Reine Angew. Math. 573(2004), 181209. MR2084587 (2005h:47014)

[GHX] K. Guo, J. Hu and X. Xu, Toeplitz algebras, subnormal tuples and rigidity on reproducing $C\left[z_{1}, \ldots, z_{n}\right]$-modules, J. Funct. Anal. 210(2004), 214-247. MR.2052120 (2005a:47007)

[GW1] K. Guo and K. Wang, Essentially normal Hilbert modules and K-homology, Math. Ann. 340(2008), 907-934. MR2372744(2009c:47006) 
[GW2] K. Guo and K. Wang, Essentially normal Hilbert modules and K-homology II: Quasihomogeneous Hilbert modules over the two dimensional unit ball, J. Ramanujan Math. Soc. 22(2007), 259-281. MR2356345 (2008h:47017)

[GWa] K. Guo and P. Wang, Essentially normal Hilbert modules and K-homology III: Homogenous quotient modules of Hardy modules on the bidisk, Sci. China Ser. A 50(2007), 387411. MR2334557 (2008j:47006)

[Hed] P. Hedenmalm, Spectral properties of invariant subspaces in the Bergman space, J. Funct. Anal. 116(1993), 441-448. MR.1239077 (95d:30071)

[Ri] S. Richter, Invariant subspaces in Banach spaces of analytic functions, Trans. Amer. Math. Soc. 304(1987), 585-616. MR911086 (88m:47056)

[Zhu] K. Zhu, Restriction of the Bergman shift to an invariant subspace, Quart. J. Math. Oxford Ser. (2) 48(1997), 519-532. MR1604847 (99a:47051)

Department of Mathematics, Southeast University, Nanjing, 210018, People's RepubLIC OF CHINA

E-mail address: 051018010@fudan.edu.cn 